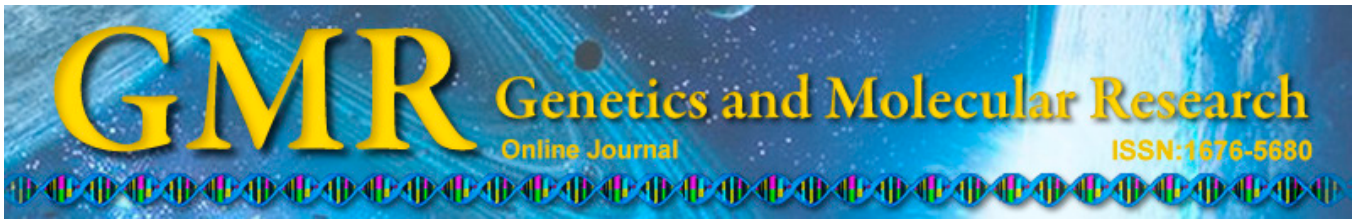

\title{
Polymorphisms in the SIRT5 gene and their association with body measurement and ultrasound traits in Qinchuan cattle
}

\author{
L.S. Gui ${ }^{1 *}$, H.C. Wang ${ }^{1 *}$, G.Y. Liu ${ }^{3}$ and L.S. Zan $^{1,2}$ \\ ${ }^{1}$ College of Animal Science and Technology, Northwest A\&F University, \\ Yangling, Shaanxi, China \\ ${ }^{2}$ National Beef Cattle Improvement Center of Northwest A\&F University, \\ Yangling, Shaanxi, China \\ ${ }^{3}$ Institute of Soil and Water Conservation, CAS\&MWR, Yangling, Shaanxi, \\ China \\ *These authors contributed equally to this study. \\ Corresponding author: L.S. Zan \\ E-mail: zanlinsen@163.com
}

Genet. Mol. Res. 14 (2): 3843-3853 (2015)

Received June 6, 2014

Accepted October 22, 2014

Published April 22, 2015

DOI http://dx.doi.org/10.4238/2015.April.22.13

\begin{abstract}
Silent information regulator 5 (SIRT5), a member of the Sirtuin family class III nicotinamide adenine dinucleotide-dependent protein deacetylases, plays an important role in metabolic and aging processes in mammals. We identified 4 single-nucleotide polymorphisms (SNPs) (G22010A, G22052A, G22119T, and G22245C) in the 3' untranslated regions of the SIRT5 gene from 572 Qinchuan cattle by sequencing and investigating their association with growth and ultrasound traits. The frequencies of genotype GG and allele $\mathrm{G}$ were high at the 4 SNPs. Based on the $\chi^{2}$ test, the genotypic distributions of the 4 SNPs were not in Hardy-Weinberg equilibrium $(\mathrm{P}<0.05$ or $\mathrm{P}<0.01)$. Association analysis of individual SNPs and haplotype combinations revealed that the 4 loci were significantly associated with some body measurement and ultrasound traits in Qinchuan cattle,
\end{abstract}


and the $\mathrm{H}_{1} \mathrm{H}_{5}$ (AG-GA-GG-GG) diplotypes had better performance than other combinations in Qinchuan cattle. Our results demonstrate that SIRT5 may be a candidate for marker-assisted selection in future breeding programs for Qinchuan cattle.

Key words: Genetic variability; Body measurement; Ultrasound; Silent information regulator 5; Single-nucleotide polymorphism

\section{INTRODUCTION}

Sirtuins are protein deacetylases that hydrolyze 1 nicotinamide adenine dinucleotide $\left(\mathrm{NAD}^{+}\right)$cosubstrate for each lysine side chain they deacetylate (Blander and Guarente, 2004). Sirtuins play a regulatory role in many biological processes, including genetic transcription, DNA repair, genome stability, and energy metabolism (Marmorstein, 2004), which has been generally attributed to their NAD-dependent deacetylase activity (Haigis and Guarente, 2006). Mammals have 7 isoforms, SIRT1-SIRT7, which have been described to have different target proteins and cellular localizations, including the cytoplasm (SIRT1 and 2), nucleus (SIRT1, 2, 3, 6, and 7), and mitochondria (SIRT3, 4, and 5). SIRT5 is the only mammalian Sirtuin grouped in subfamily III, which includes mainly prokaryotic sirtuins (Gertz and Steegborn, 2010).

SIRT5 is an endogenous protein localized in the mitochondrial matrix (Hirschey, 2011) and is primarily expressed in the brain, muscle, heart, liver, and kidney tissues (Michishita et al., 2005). A number of research studies have suggested that the SIRT1 and SIRT2 genes enhance proliferation of preadipocytes and inhibit preadipocyte apoptosis through their acetylation/deacetylation functions (Bai et al., 2007; Wang and Tong, 2009). Similarly, recent studies have suggested that SIRT5 has a similar function in humans (Nakagawa, 2009), but its mechanism of action remains unclear. In addition, SIRT5 interacts with carbamoyl phosphate synthetase 1, which catalyzes the first step of ammonia detoxification and disposal via the urea cycle. It is possible that SIRT5 stimulates the deacetylation function of carbamoyl phosphate synthetase 1with $\mathrm{NAD}^{+}$in vitro (Schuetz et al., 2007). Lysine succinylation has been consistently shown to be a new posttranslational modification and SIRT5 serves as a key enzyme for removing succinyl groups from lysine residues of multiple cellular proteins (Nakagawa et al., 2009). Based on its localization and biological functions, these findings indicate that SIRT5 links metabolic and aging processes in mammals.

However, studies of polymorphisms in the SIRT5 gene have not been reported. Therefore, we estimated genetic variations in the SIRT5 gene in 572 Qinchuan cattle using DNA sequencing methods and determined the association between its quantitative traits loci and body measurement and ultrasound traits. Our results are useful for further studies of the SIRT5 gene.

\section{MATERIAL AND METHODS}

Animal handling procedures were performed in accordance with the Guide for the Care and Use of Laboratory Animals, and the animal study protocol was approved by the Institutional Animal Care and Use Committee of Northwest A\&F University. 


\section{Genomic DNA preparation and phenotypic data collection}

A total of 572 adult animals were randomly selected from Qinchuan cattle breeding populations (without genetic relationships), and stratified into age categories of 18-24 months. DNA samples were extracted from blood samples and then stored at $-80^{\circ} \mathrm{C}$ (Sambrook et al., 1989). DNA content was estimated spectrophotometrically, and then the genomic DNA was diluted to $50 \mathrm{ng} / \mathrm{L}$. All DNA samples were stored at $-20^{\circ} \mathrm{C}$ until subsequent analysis.

For further association studies, body measurement traits, including body length (BL), withers height (WH), hip height (HH), rump length (RL), hip width (HW), chest depth (CD), chest circumference (CC), and pin bone width (PBW), were measured as described previously (Gilbert et al., 1993). Ultrasound traits, including backfat thickness (BT), ultrasound loin muscle area (ULA), and intramuscular fat (IF), were measured using the Rincon method (Rincon et al., 2009). To reduce systematic error, a single person measured each of the 11 traits in all animals.

\section{Primer design and polymerase chain reaction (PCR) conditions}

Primers to amplify of the bovine SIRT5 gene were designed based on sequences in the NCBI database (GenBank accession No. NP_001029467.1) using the Primer v5.0 software (PREMIER Biosoft, Palo Alto, CA, USA). Forward primer 3'-Region-F (5'-TGCTGTGTTCG TCCTTCTGT-3') and reverse primer 3'-Region-R (5'-CTATCCCCACCCCGAACTT-3') were used to amplify a fragment of 582 base pair in the 3 ' untranslated region (UTR) of the SIRT5 gene. PCR was conducted in $20-\mu \mathrm{L}$ reactions containing 50 ng DNA, $10 \mathrm{pM}$ of each primer, $0.20 \mathrm{mM}$ dNTPs, $2.5 \mathrm{mM} \mathrm{MgCl}$, and 0.5 U Taq DNA polymerase (TaKaRa, Shiga, Japan). The following PCR reaction conditions were used: $5 \mathrm{~min}$ at $95^{\circ} \mathrm{C} ; 35$ cycles of $30 \mathrm{~s}$ at $94^{\circ} \mathrm{C}$, $35 \mathrm{~s}$ at $58.7^{\circ} \mathrm{C}, 40 \mathrm{~s}$ at $72^{\circ} \mathrm{C}$, and final extension at $72^{\circ} \mathrm{C}$ for $10 \mathrm{~min}$. Digested products were detected by electrophoresis on $1.5 \%$ agarose gels.

\section{Genotyping of SIRT5 alleles by sequencing}

Using single-strand conformation polymorphism to detect the 4 mutations would be time-consuming and very complicated. Additionally, there are no suitable restriction endonucleases for restriction fragment length polymorphism, which is commonly used to detect mutation genotypes. Thus, DNA from the 572 Qinchuan cattle were sequenced to distinguish the genotypes of the 4 mutations in SIRT5.

\section{Statistical analysis}

Gene frequencies, allelic frequencies, and Hardy-Weinberg equilibrium were evaluated by direct counting. Population genetic indices including gene heterozygosity $\left(H_{\mathrm{E}}\right)$, gene homozygosity $\left(H_{\mathrm{O}}\right)$, effective allele numbers $\left(N_{\mathrm{E}}\right)$, and polymorphism information content (PIC) were calculated according to Nei's methods (Nei and Roychoudhury, 1974). Linkage disequilibrium and haplotype distribution of the SNPs were analyzed using the expectation maximization algorithm with Haploview software (Barrett et al., 2005).

SPSS v. 13.0 (SPSS, Inc., Chicago, IL, USA) was used to analyze the relationship between different genotypes of the SIRT5 gene, body measurement traits (BL, WH, HH, RL, 
HW, CD, CC, and PBW), and ultrasound traits (BT, ULA, and IF) in Qinchuan cattle. The following statistical linear model was used: $Y i j=\mu+G i+A i+E i j k$, where $Y i j$ was the traits measured on each of the individual cattle, $\mu$ was the overall population mean for the traits, $G i$ was the fixed effect associated with the genotype, $A_{\mathrm{i}}$ was the fixed effect due to age, and $E_{\mathrm{ijk}}$ was the standard error.

\section{RESULTS}

\section{Polymorphisms and genetic diversity}

Four polymorphic sites in the SIRT5 gene (Figures 1-4), including G22010A, G22052A, G22119T, and G22245C, located in 3'UTR of SIRT5 were identified by sequencing. Data showed that both G22119T and G22245C had 2 genotypes and the TT and CC genotypes were not observed in the animals sampled. Genotype and allele frequencies for the 4 loci are shown in Table 1. The G allele was predominant at the 4 SNPs. According to the $\chi^{2}$ test, the genotypic distributions of 4 SNPs were not in Hardy-Weinberg equilibrium $(\mathrm{P}<0.05$ or $\mathrm{P}<0.01)$.

According to Nei's methods, population genetic indices, including $H_{\mathrm{E}}, N_{\mathrm{E}}$, and PIC, were calculated and the results are shown in Table 1. The PIC value is an effective indicator of genetic diversity from different loci of the candidate gene. Our results showed that both G22010A and G22052A had medium polymorphism levels $(0.250<\mathrm{PIC}<0.500)$, whereas G22119T and G22245C had low polymorphism levels (0.250 > PIC).

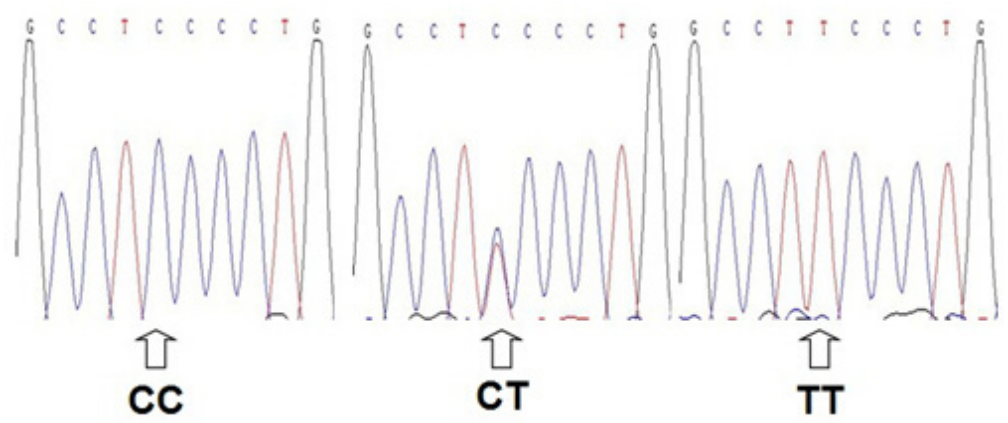

Figure 1. Sequencing map of the SNP22010 locus (reverse direction).

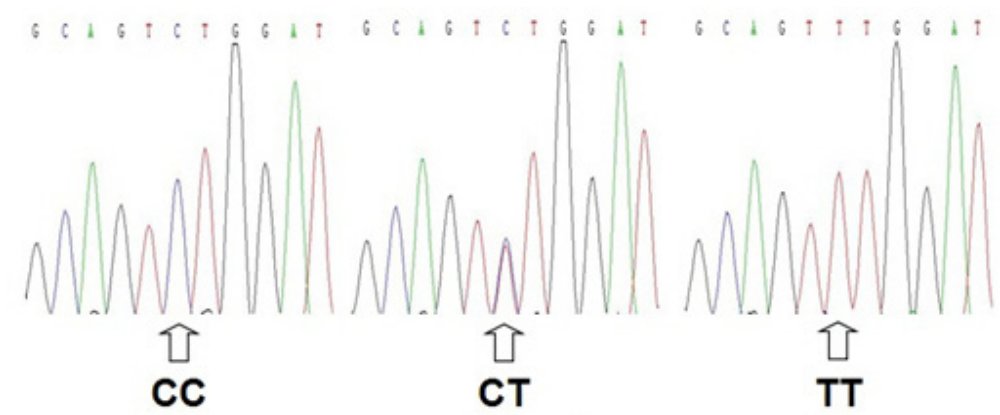

Figure 2. Sequencing map of the SNP22052 locus (reverse direction). 


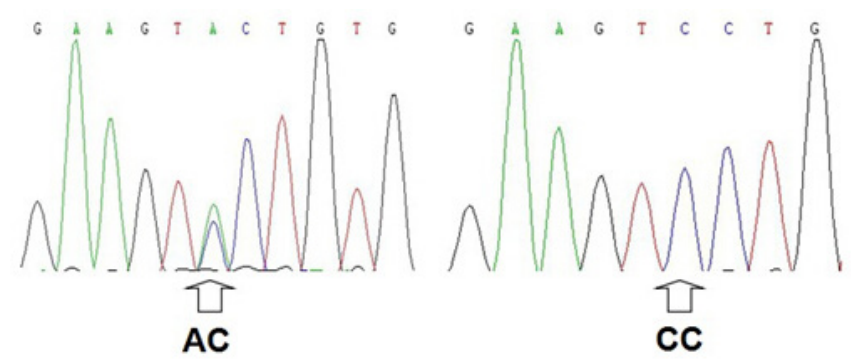

Figure 3. Sequencing map of the SNP22119 locus (reverse direction).

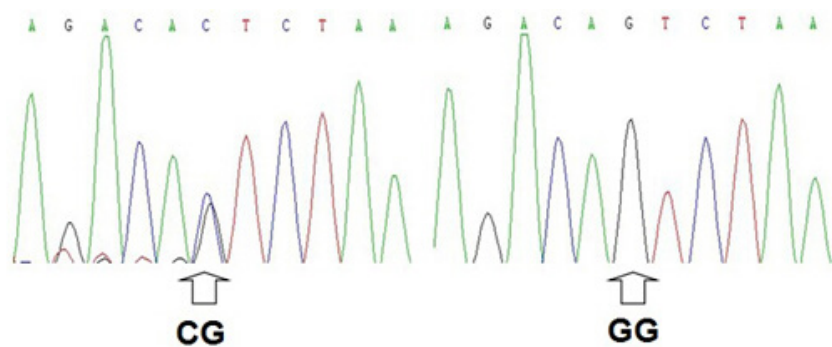

Figure 4. Sequencing map of the SNP22245 locus (reverse direction).

Table 1. Genotype frequencies (\%) of the SIRT5 gene for SNPs in Qinchuan cattle populations.

\begin{tabular}{|c|c|c|c|c|c|c|c|c|c|c|}
\hline Site & Sample & & typic frec & & Allele & uency & $H_{\mathrm{E}}$ & $N_{\mathrm{E}}$ & PIC & $\chi^{2}$ (HWE*) \\
\hline G22010A & 572 & $\begin{array}{c}\text { GG } \\
0.5769\end{array}$ & $\begin{array}{c}\text { GA } \\
0.3357\end{array}$ & $\begin{array}{c}\mathrm{AA} \\
0.0874\end{array}$ & $\begin{array}{c}\mathrm{G} \\
0.7448\end{array}$ & $\begin{array}{c}\mathrm{A} \\
0.2552\end{array}$ & 0.3802 & 1.6134 & 0.3079 & 7.8453 \\
\hline G22052A & 572 & $\begin{array}{c}\text { GG } \\
0.5664\end{array}$ & $\begin{array}{c}\mathrm{AG} \\
0.3322\end{array}$ & $\begin{array}{c}\text { AA } \\
0.1014\end{array}$ & $\begin{array}{c}\mathrm{G} \\
0.7325\end{array}$ & $\underset{0.2675}{\mathrm{~A}}$ & 0.3919 & 1.6444 & 0.3151 & 13.2772 \\
\hline $\mathrm{G} 22119 \mathrm{~T}$ & 572 & $\begin{array}{c}\text { GG } \\
0.7762\end{array}$ & $\begin{array}{c}\text { GT } \\
0.2238\end{array}$ & 0 & $\begin{array}{c}\mathrm{G} \\
0.8881\end{array}$ & $\begin{array}{c}\mathrm{T} \\
0.119\end{array}$ & 0.1987 & 1.2480 & 0.1790 & 9.0788 \\
\hline G22245C & 572 & $\begin{array}{c}\text { GG } \\
0.7972\end{array}$ & $\begin{array}{c}\mathrm{GC} \\
0.2028\end{array}$ & 0 & $\begin{array}{c}\mathrm{G} \\
0.8986\end{array}$ & $\begin{array}{c}\mathrm{C} \\
0.1014\end{array}$ & 0.1822 & 1.1228 & 0.1656 & 7.2833 \\
\hline
\end{tabular}

$\mathrm{HWE}=$ Hardy-Weinberg equilibrium; $\chi 0.05^{2}=5.991, \chi 0.01^{2}=9.210$.

\section{Linkage disequilibrium and haplotype analysis}

Linkage disequilibrium between polymorphism pairs and haplotype structure analyses of the SIRT5 gene are shown in Table 2 and 3. To determine linkage relationships among the 4 SNPs, the linkage disequilibrium between the 4 sites was estimated. The results indicated that the $r^{2}$ values ranged from 0.000-1.000. According to a previous study, if the value of $r^{2}$ is over 0.33, linkage disequilibrium is considered to be strong (Ardlie et al., 2002). Our results revealed strong linkage between G22052A and G22245C, while others linkages with pair-wise $r^{2}<0.33$ were weak.

Haplotype analyses for the 4 SNPs showed that 5 different haplotypes were present in the populations studied, with frequencies greater than 0.05 (haplotypes with frequency $<0.05$ were ignored). Hap2 (-GGGG-) showed the highest haplotype frequencies $(41.30 \%)$, followed by Hap5 (-GAGG-), and Hap1 (-AGGG-). The high-frequency haplotypes were likely been 
present in the population for a long time, which may be directly or indirectly regulated by different rearing environments (Gui et al., 2015).

Table 2. Estimated values of linkage disequilibrium for SNPs in bovine SIRT5 of Qinchuan cattle.

\begin{tabular}{lcccc}
\hline SNP & G22010A & G22052A & G22119T & G22245C \\
\hline G22010A & - & $\mathrm{D}=0.220$ & $\mathrm{D}=0.042$ & $\mathrm{D}=0.033$ \\
G22052A & $r^{2}=0.006$ & - & $\mathrm{D}=0.455$ & $\mathrm{D}=0.961$ \\
G22119T & $r^{2}=0.000$ & $r^{2}=0.009$ & $r^{2}=0.009$ & $\mathrm{D}=0.102$ \\
G22245C & $r^{2}=0.000$ & $r^{2}=0.038$ & - \\
\hline
\end{tabular}

Table 3. Haplotypes of SIRT5 gene and their frequencies in Qinchuan cattle.

\begin{tabular}{lccccc}
\hline Haplotype & G22010A & G22052A & G22119T & G22245C & Frequency \\
\hline Hap1 & G & G & G & G & 0.167 \\
Hap2 & G & G & G & G & 0.413 \\
Hap3 & G & G & G & G & 0.059 \\
Hap4 & G & G & T & G & 0.060 \\
Hap5 & G & G & G & G & 0.187 \\
\hline
\end{tabular}

\section{Effects of single markers/haplotype combinations on growth traits and ultrasound traits in Qinchuan cattle}

Table 4 shows the effects of the SNPs on growth performance and ultrasound in Qinchuan cattle. The 4 polymorphisms mainly affected cattle body measurement traits and ultrasound trait. At the SNP22010 locus, individuals with genotype GG had higher values than those with GA for WH, HH, RL, and CC $(\mathrm{P}<0.05)$. Additionally, the $\mathrm{HW}$ and PBW of individuals with genotype AA were higher than those with genotype AG $(\mathrm{P}<0.01)$. At the SNP22052 locus, individuals with genotype AG had higher values than those with AA on $\mathrm{BL}, \mathrm{WH}, \mathrm{RL} \mathrm{CD}$, and $\mathrm{CC}(\mathrm{P}<0.05)$, while genotype $\mathrm{AG}$ had higher mean values for $\mathrm{WH}$, $\mathrm{HH}, \mathrm{CC}$, and ULA than those with genotype GG $(\mathrm{P}<0.05)$. Significant differences in HW and PBW were observed between the AG and GG genotypes $(\mathrm{P}<0.01)$. Compared with AA, individuals with the AG genotype showed better performance for $\mathrm{HH}$ and $\mathrm{PBW}(\mathrm{P}<0.01)$. At the SNP22119 locus, individuals with GG genotype had very significantly $(\mathrm{P}<0.05)$ greater values of BL, RL, HW, CC, and IF than those with the GT genotype. At the SNP22245 locus, significant differences in BL, HH, RL, CD, PBW, and IF were observed between the GG and $\mathrm{GC}$ genotypes $(\mathrm{P}<0.05)$. However, no significant correlations were observed in the remaining indices for the 4 SNPs.

Multiple effects of the 4 SNPs were evaluated and a total of 18 haplotype combinations were identified. Combinations with frequencies lower than 5.0\% (data not shown) were not included and the remaining combinations were further analyzed. In Table 5, individuals with $\mathrm{H}_{1} \mathrm{H}_{5}$ and $\mathrm{H}_{2} \mathrm{H}_{2}$ showed significantly higher $\mathrm{HW}$ than those with $\mathrm{H}_{1} \mathrm{H}_{2}(\mathrm{P}<0.05)$. Individuals with diplotypes $\mathrm{H}_{1} \mathrm{H}_{5}$ had higher values than those with $\mathrm{H}_{1} \mathrm{H}_{2}$ and $\mathrm{H}_{4} \mathrm{H}_{5}$ for $\mathrm{CC}(\mathrm{P}<0.05)$, and similar results were observed between $\mathrm{H}_{1} \mathrm{H}_{2}$ and $\mathrm{H}_{5} \mathrm{H}_{5}(\mathrm{P}<0.01)$. In addition, individuals with $\mathrm{H}_{1} \mathrm{H}_{5}$ exhibited significantly larger $\mathrm{PBW}$ than those with $\mathrm{H}_{1} \mathrm{H}_{2}(\mathrm{P}<0.01)$. For ultrasound traits, individuals with the combined genotype $\mathrm{H}_{1} \mathrm{H}_{5}$ showed a significantly difference than the others $(\mathrm{P}<0.01)$ for ULA. $\mathrm{H}_{4} \mathrm{H}_{5}$ had the lowest IF value compared with the other diplotypes $(\mathrm{P}<0.01)$. 


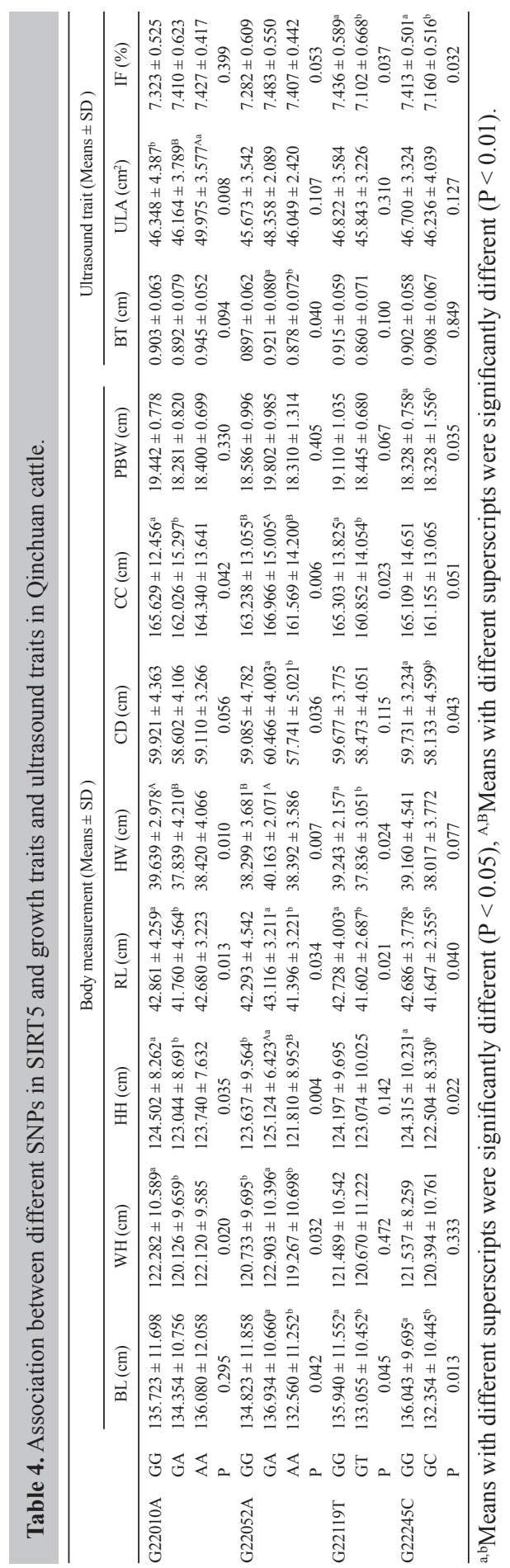




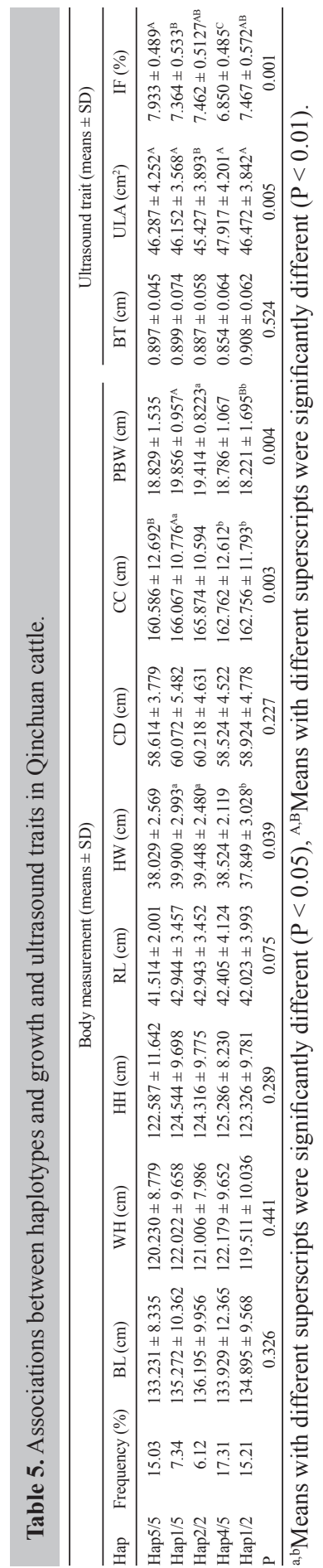




\section{DISCUSSION}

Mitochondria are essential organelles in cellular energy metabolism because they supply the cell with metabolic energy in the form of ATP generated by oxidative phosphorylation (Martinou and Youle, 2011). They are also important in processes such as apoptosis, cellular senescence, and lifespan regulation (Balaban et al., 2005). Among the mammalian sirtuins gene, SIRT5 is located in the mitochondrial matrix and is the only protein shown to possess efficient demalonylase and desuccinylase activity (Du et al., 2011). Schlicker et al. (2008) reported that the SIRT5 gene could deacetylate cytochrome c, a protein in the mitochondrial intermembrane space with a central function in oxidative metabolism and apoptosis. A previous proteomic survey verified that lysine succinylation is a new posttranslational modification in proteins using Escherichia coli extracts (Zhang et al., 2011), and Peng et al. (2011) reported that SIRT5 served as a key enzyme that removed succinyl groups from the lysine residues of multiple cellular proteins. Because of its location and its function in the mitochondria, we hypothesized that the SIRT5 gene is associated with cattle body measurement and ultrasound traits.

To evaluate the effects of the 4 SNPs in the SIRT5 gene on growth traits and ultrasound traits in Qinchuan cattle, the association between SIRT5 polymorphisms and 11 traits, including 8 body measurement traits and 3 ultrasound traits, were examined. Our results showed that the AA genotypes (G22010A), GG genotypes (G22052A), AG genotypes (G22119T), and GG genotypes (G22245C) were highly associated growth traits and ultrasound traits compared to other genotypes in single analysis. Furthermore, we examined the association between haplotype combinations and the above traits. The diplotypes $\mathrm{H}_{2} \mathrm{H}_{2}$ (GG-GG-GG-GG), $\mathrm{H}_{1} \mathrm{H}_{5}$ (GA-GA-GG-CG), and $\mathrm{H}_{1} \mathrm{H}_{2}$ (GA-GG-GG-GG) showed higher associations than the other haplotype combinations. Compared with the combination results, individuals with the $\mathrm{H}_{2} \mathrm{H}_{2}$ haplotype showed better performance for growth and ultrasound.

The 4 SNPs identified in this study were located in the 3'UTR region, and did not change the structure of the encoded proteins; however, the 3'UTRs of eukaryotic genes were reported to regulate mRNA stability, localization, and translation, and the molecular genetic variability of the $3^{\prime}$ region sequence or its binding and splicing factors may be responsible for these modifications (Rigo et al., 2008). Previous reports showed that mutations in the 3'UTR could affect gene expression, phenotype, and consequently respective physiological functions. For instance, Yie et al. (2008) identified a single base pair mutation in the human leukocyte antigen-G 3'UTR, which was thought to be a fundamental mechanism for lowering the levels of placental human leukocyte antigen-G protein expression in patients with preeclampsia. Zhou et al. (2011) identified an SNP in the malic enzyme 1 gene 3'UTR, and showed that it was correlated with ultrasound in Chinese red cattle.

\section{CONCLUSIONS}

In summary, we reported polymorphisms in the SIRT5 gene in Qinchuan cattle and demonstrated an association between the SIRT5 gene and body measurement and ultrasound traits. Our data strongly suggest that the combined genotype $\mathrm{H}_{2} \mathrm{H}_{2}$ can be used as a genetic marker for the selection and breeding of Qinchuan. Our results suggest that the SIRT5 gene can be used as a candidate gene for breeding. Further studies should be conducted in a large population before using this gene for molecular marker-assisted selection. 


\section{ACKNOWLEDGMENTS}

Research supported by the National Science \& Technology Pillar Program during the 12th Five-year Plan Period (\#2011BAD28B04-03), the State Natural Sciences Foundation (\#31272411), the National Beef and Yak Industrial Technology System (\#CARS-38), the China National "863" Program (\#2013AA102505), and the Major Science and Technology Program of Xinjiang Construction Corps (\#2014AA001-1).

\section{REFERENCES}

Ardlie KG, Kruglyak L and Seielstad M (2002). Patterns of linkage disequilibrium in the human genome. Nat. Rev. Genet. 3: 299-309.

Bai L, Pang WJ, Yang YJ and Yang GS (2007). Modulation of SIRT1 by resveratrol and nicotinamide alters proliferation and differentiation of pig preadipocytes. Mol. Cell. Biochem. 307: 129-140.

Balaban RS, Nemoto S and Finkel T (2005). Mitochondria, oxidants, and aging. Cell 120: 483-495.

Barrett JC, Fry B, Maller J and Daly MJ (2005). Haploview: analysis and visualization of LD and haplotype maps. Bioinformatics 21: 263-265.

Blander G and Guarente L (2004). The Sir2 family of protein deacetylases. Annu. Rev. Biochem. 73: 417-435.

Du J, Zhou Y, Su X, Yu JJ, et al. (2011). Sirt5 is an NAD-dependent protein lysine demalonylase and desuccinylase. Science 334: 806-809.

Gertz M and Steegborn C (2010). Function and regulation of the mitochondrial Sirtuin isoform Sirt5 in Mammalia. Biochim. Biophys. Acta 8: 1658-1665.

Gilbert RP, Bailey DR and Shannon NH (1993). Linear body measurements of cattle before and after 20 years of selection for post weaning gain when fed two different diets. J. Anim. Sci. 71: 1712-1720.

Gui L, Jiang B, Zhang Y and Yan L (2015). Sequence variant in the bovine silent information regulator 6, their linkage and their associations with body measurements and carcass quality traits in Qinchuan cattle. Gene 559: 16-21.

Haigis MC and Guarente LP (2006). Mammalian sirtuins-emerging roles in physiology, aging, and calorie restriction. Genes Dev. 20: 2913-2921.

Hirschey MD (2011). Old enzymes, new tricks: sirtuins are NAD+-dependent deacylases. Cell Metab. 14: 718-719.

Marmorstein R (2004). Structure and chemistry of the Sir2 family of $\mathrm{NAD}^{+}$-dependent histone/protein deactylases. Biochem. Soc. Trans. 32: 904-909.

Martinou JC and Youle RJ (2011). Mitochondria in apoptosis: Bcl-2 family members and mitochondrial dynamics. Dev. Cell 21: 92-101.

Michishita E, Park JY, Burneskis JM, Barrett JC, et al. (2005). Evolutionarily conserved and nonconserved cellular localizations and functions of human SIRT proteins. Mol. Biol. Cell 16: 4623-4635.

Nakagawa T, Lomb DJ, Haigis MC and Guarente L (2009). SIRT5 deacetylates carbamoyl phosphate synthetase 1 and regulates the urea cycle. Cell 137: 560-570.

Nei M and Roychoudhury AK (1974). Sampling variance of heterozygosity and genetic distance. Genetics. 76: 379-390.

Peng C, Lu Z, Xie ZY, Cheng ZY, et al. (2011). The first identification of lysine malonylation substrates and its regulatory enzyme. Mol. Cell. Proteomics Epub ahead of print.

Rigo F and Martinson HG (2008). Functional coupling of last-intron splicing and 3'-end processing to transcription in vitro: the poly (A) signal couples to splicing before committing to cleavage. Mol. Cell Biol. 28: 849-862.

Rincon G, Farber EA, Farber CR, Nkrumah JD, et al. (2009). Polymorphisms in the STAT6 gene and their association with carcass traits in feedlot cattle. Anim. Genet. 40: 878-882.

Sambrook J, Fritsch EF and Maniatis T (1989). Molecular cloning: a laboratory manual. Appendix E3. 2nd edn. Cold Spring Harbor Laboratory, Cold Spring Harbor, NY.

Schlicker C, Gertz M, Papatheodorou P, Kachholz B, et al. (2008). Substrates and regulation mechanisms for the human mitochondrial sirtuins Sirt3 and Sirt5. J. Mol. Biol. 3: 790-801.

Schuetz A, Min J, Antoshenko T, Wang CL, et al. (2007). Structural basis of inhibition of the human NAD ${ }^{+}$-dependent deacetylase SIRT5 by suramin. Structure 15: 377-389.

Wang F and Tong Q (2009). SIRT2 suppresses adipocyte differentiation by deacetylating FOXO1 and enhancing FOXO1's repressive interaction with PPARgamma. Mol. Biol. Cell 20: 801-808.

Yie S, Li L, Xiao R and Librach CL (2008). A single base-pair mutation in the 3'-untranslated region of HLA-G mRNA is 
associated with preeclampsia. Mol. Hum. Reprod. 14: 649-653.

Zhang ZH, Tan MJ, Xie ZY, Dai LZ, et al. (2011). Identification of lysine succinylation as a new post-translational modification. Nat. Chem. Biol. 7: 58-63

Zhou GL, Caob Y and Jin HG (2011). Cloning and polymorphisms of the 3'-untranslated region of malic enzyme gene in Chinese red cattle. Meat Sci. 89: 72-75. 